\title{
Coordinating standardization in Dynamic Spectrum Access
}

\author{
Simon Delaere, Vânia Gonçalves \\ iMinds-SMIT, Vrije Universiteit Brussel \\ Brussels, Belgium \\ firstname.lastname@vub.ac.be
}

\author{
Paulo Marques \\ Instituto de Telecomunicações \\ Aveiro, Portugal \\ pmarques@av.it.pt
}

\begin{abstract}
This paper investigates the gap between research into Dynamic Spectrum Access (DSA), and the standardization of these technologies, as well as ways in which coordination of standardization activities between $\mathrm{EU}$ funded research projects might help bridge this gap. Concretely, it first discusses the current state of standardization in CR and DSA, as well as the need for a coordinated approach to DSA standardization among European projects. It subsequently provides a short overview of the DSA and CR research landscape in Europe and briefly describes the objectives of the Cognitive Radio Standardization Initiative (CRS-i). The following sections on the paper then focus on a survey which was conducted among the 26 currently funded, Radio Access and Spectrum related EU projects, with specific analysis also performed for the CR and DSA related sub-cluster of projects. This survey provides insight into projects' current standardization activities and collaboration methods, as well as opportunities and bottlenecks for collaboration in standardization. The paper concludes that the coordination of standardization efforts, as well as specific instruments to enable the standardization of research results beyond the project lifetime, are desirable if Europe is to play a role in the global standardization of CR and DSA. Based on the survey and on close interactions with RAS cluster projects, the paper finally proposes a number of concrete coordination trajectories.
\end{abstract}

Keywords: Dynamic Spectrum Access, Cognitive Radio, Standardization, coordination, EU Research, FP7, survey

\section{INTRODUCTION}

Over the past decade, cognitive radio (CR) systems for Dynamic Spectrum Access (DSA) have been seen as a candidate technology to provide the necessary capacity in otherwise rather congested useful frequency bands. Research into this topic has matured and many projects and initiatives have provided proof of concept implementations, demonstrators and showcases. However, despite several years of activities aimed at bringing project results to standardization, there are only few standards that may be used as benchmark for the type approval or the certification of the operation of CR equipment and they are rather incomplete and rather fragmented. One major reason for this is the significant gap that still exists between European research into Dynamic Spectrum Access and the standardization of these technologies. Such gap is in its turn caused by the fact that projects face difficulties in achieving impact during their rather short lifetime. Moreover, there is often a time mismatch between the project timeline and the roadmap of a particular standardization initiative. Partly because of this, both Japan, the US and China are currently leading the standardization activities on cognitive radio and DSA.

Since a few years, it has been understood that a more collaborative approach is required between research projects in order to increase the transfer of promising EU research results to standardization. Specifically for the field of DSA and CR research, the Cognitive Radio Standardization initiative (CRS-i) was launched at the end of 2012 with the objective of coordinating and supporting existing and future FP7 projects and of facilitating the exploitation of their results by establishing a concentrated approach to Cognitive Radio Systems standardization. The initiative intends to function as the primordial means of communication between a cluster of currently funded DSA-related projects. In order to prepare for this interaction, a survey was set up in order to understand their current standardization activities and collaboration methods, as well as to identify opportunities and bottlenecks for collaboration in standardization. The survey was conducted among all 26 projects of the Radio Access and Spectrum (RAS) cluster [1], an initiative of the European 7th Framework Programme, and specific analysis was also performed for the CR and DSA related sub-cluster of projects. The results of the survey are described in this paper. It is structured as follows: Section II discusses the current state of standardization in CR and DSA, as well as the need for a coordinated approach to DSA standardization among European projects. It also provides a short overview of the DSA and CR research landscape at the time of writing of the paper and briefly describes the objectives of CRS-i and how the survey that was carried out fits with these objectives. Section III then explains the set-up of the survey, while Section IV provides an overview of the results obtained. Finally, Section V provides a discussion of results, in particular with regard to the opportunities and bottlenecks of standardization coordination. The section subsequently proposes a number of concrete recommendations and proposals for coordinated standardization between RAS cluster projects. 


\section{BRIDGING THE STANDARDIZATION GAP IN DSA RESEARCH}

\section{A. Standards in CR and DSA}

Research into cognitive radio systems and cognitive radio networks has matured and many projects and initiatives have provided proof of concept implementations, demonstrators and showcases. In the military domain first products are shipped on the market. However, these vertical market products are not following widely agreed standards and there are no commonly approved testing and certification mechanisms in place. The WInnF (Wireless Innovation Forum, formerly SDR Forum) has developed standard like documents, the CEPT's CAM (Telecommunications Conformity Assessment and Market Surveillance Committee) has discussed requirements and regulatory implications, the national regulators have issued their ideas, or dockets, of proposed rulemaking to govern the use of cognitive radio systems. But to date, there are only few standards that may be used as benchmark for the type approval or the certification of the operation of CR equipment and they are rather incomplete and rather fragmented. Notably, there is a range of standards in the IEEE 802 family, in the DySPAN (formerly SCC41) 1900.x family and significant efforts by the ETSI RRS technical committee and related working groups. Specifically in the EU, CR/SDA standardization received a new impetus as the European Commission, after having organized a workshop in Ispra (Italy) on Software Defined Radio and Cognitive Radio standardization in November 2011 [2] formally submitted a standardization mandate to CEN, CENELEC and ETSI for Reconfigurable Radio Systems in October 2012 [3]. Final results of this mandate are expected by the Commission to come within 42 months after its acceptance (which was reported in May 2013). Within ETSI, work on the mandate is currently divided over different Technical Committees: while ETSI TC RRS (Technical Committee on Reconfigurable Radio Systems) is responsible for ETSI work on the Mandate, ETSI TC BRAN (Technical Committee on Broadband Radio Access Networks) had already started working on a Harmonized Standard before the Mandate was issued and is continuing this work; and ETSI TC RRS and TC ERM (Technical Committee on Electromagnetic Compatibility and Radio Spectrum Matters) have also agreed to create a task force (JTFER) with the aim of developing RRS related Harmonised Standards (including the Harmonised Standards developed in response to the mandate). At the time of writing of the paper, ongoing commercial sector oriented RRS work in relation to the mandate focuses on four technical specification documents, dealing with various aspects of broadband operation in TV White Spaces and the functioning of Geolocation Databases. Potential future work could include the use of Cognitive Radio Systems in other frequencies and under an LSA (License Shared Access) regime amongst other things. [4]

\section{B. The need for a coordinated approach to DSA standardization}

In parallel to the standardization activities mentioned above, a range of projects have been funded as part of the FP6 and FP7 programmes, investigating the theoretical aspects of cognitive radios, developing demonstrators and producing contributions to one or the other standardisation effort. Similarly, in the USA, NSF funded projects along with initiatives led by Motorola, Microsoft, or Google have reached noteworthy outcomes. In Japan, a common approach to CR systems was investigated as part of a national initiative, driven by IEICE, on cognitive radio research.

As indicated in [5], [6] and [8], standards are needed to close the gap between research outcomes and commercial exploitation. However, when considering all the studies mentioned (particularly the FP7 projects), and looking at the amount of project outcomes and the individual rather small scale standardisation efforts and fragmented standardisation outcomes, there still seems to be a significant gap between research and standardization. Standardisation is only a side aspect in research projects, even though most related FP7 projects do participate in and contribute to standardisation bodies. However, all projects face the fact that it is difficult to achieve impact during the rather short (typically 2-3 years) life time of an FP7 project; standardization is a relatively slow process and therefore needs a more continuous engagement than a 36 month project can provide. Therefore, there is a large risk that the outcomes of FP7 projects will have little impact on the work being carried by standardization bodies, as once the project ends, there is only limited and noncoordinated transfer of the standardization inputs these projects make (and have made). Projects in general end exactly at the point in time when they are ready to produce relevant and mature input to standardization bodies. If project partners continue active in those bodies beyond the duration of project, there is a limited chance that they could still push project results towards standardisation.

Partly because of this, both Japan and US (and increasingly, China) are leading the standardization activities on cognitive radio; they drive the development in the 802.xx and 1900.x families and (with China) are also very present within ETSI RRS. Furthermore, compared to the (geographically) wide open spaces in US, and the homogeneous regulatory structure in both, US and Japan, CR in Europe has specific requirements (for example, in terms of the geo-location database, the datasets proposed by FCC and by UK-Ofcom for TV white space operation are quite different). FCC divides white space devices into two categories-"Mode I" and "Mode II" and standards such as the IETF PAWS (Protocol to Access the Whitespace database) have been designed to map the FCC requirements. These specific requirements, which are often misunderstood, bring non-European standards to develop solutions for their 'ecosystems', leaving Europe lagging behind. This acts as a powerful brake to the adoption of CR technologies in Europe, specifically because there is a high expectation on standards to accommodate various environments as experienced in Europe.

Several initiatives have already been taken in Europe to discuss and address the need of standardization collaboration between projects. The FP7 Future Networks 7th Concertation Plenary Meeting that took place in Brussels on 10 February 2011, under the topic "From Research to Innovation via Standardisation" concluded that barriers remain to the efficient participation of FP7 projects in the standardization process. The European Commission stated at the time that it aimed to 
facilitate a more systematic policy driven approach, coupling R\&D with standards [9]. Meanwhile, the Future Internet Assembly (FIA) launched a pre-standardization work group on the action of "closing the pre-standardization gap", across all of the Commission's network and service oriented projects [6].

Although research projects have contributed to $\mathrm{CR}$ standardization, barriers still remain to the efficient participation of FP7 projects in the standardization process such as: confidentiality; IPR or membership of a standards organization; synchronizing research activities with standards work and standards life-cycle; or finding the standards and standards organizations most relevant to a project, and contacting them. In particular, the CR standardization environment is a dynamic environment with several standards bodies and many working groups, making it relatively hard for research projects finding the organization(s) and particular working group that best fit their needs and objectives. What further complicates and slows down the process of $\mathrm{CR}$ Standardisation is the fact that this technology envisages a new use of radio spectrum which has to convince not only the standards committees (e.g. ETSI) but also regulators (e.g. CEPT).

\section{CRS-I: A COORDINATED R\&D EFFORT TOWARDS CR STANDARDIZATION}

In this context, a Coordination Action called Cognitive Radio Standardization initiate (CRS-i) funded by the EC started in November 2012 [21]. The scope of CRS-i is to coordinate and support existing and future FP7 projects and to facilitate the exploitation of their results by establishing a concentrated approach to Cognitive Radio Systems standardization. An important instrument implemented by CRS-i is a Standardization Consultancy Service that will strengthen the position of FP7 projects in standardization bodies related to cognitive radio at a global level, and will facilitate a coherent standardization approach in order to avoid fragmentation of standardization effort.

CRS-i operates within the framework of the Radio Access and Spectrum (RAS) cluster comprising a portfolio of more than 20 research projects participating in the 7th Framework Program (Objective 1.1 - Future Networks) and investigating Radio Access and Spectrum aspects of future wireless networks. Besides having taken responsibility of the RAS cluster and helping to formulate the cluster's Rolling Work Programme, CRS-i deploys coordination activities to a specific subset of DSA-related projects; due to space constraints, we refer to the CRS-i website for an overview of these projects. [7]. In order to prepare for these coordination activities, it was important to understand the projects' current standardization activities and collaboration methods, as well as to identify opportunities and bottlenecks for collaboration in standardization. To this end, an on-line questionnaire was set up. The answers obtained were subsequently used to organise more tailored, peer-to-peer interactions with projects in the framework of the CRS-i standardization consultancy service, as well as discussions in 1) the RAS cluster, 2) International Advisory Board interactions and/or 3) dedicated workshops. The following sections of this paper will describe the set-up and main results of the survey, and the proposals for coordination that have followed from the survey and interactions with projects, notably at meetings with the RAS projects in October 2013 (Concertation meeting), March 2014 (FIA Athens Workshop) and June 2014 (EUCNC workshop).

\section{THE SURVEY: METHOdOLOGY}

First of all, it is important to note that the survey was directed at all ongoing and recently funded Radio Access and Spectrum related projects in the EU's $7^{\text {th }}$ Framework Programme (26 projects in total) and therefore not only at the 11 DSA-related projects. The reason for this is threefold. Firstly, surveying a larger number of projects significantly increases the validity of results obtained, particularly since it can be assumed that project standardization as well as standardization coordination opportunities and bottlenecks will be largely similar for DSA and non-DSA spectrum related projects. Secondly, since CRSi took up the chairmanship of the RAS cluster, it was deemed logical to provide this particular service to a wider array of projects than only the DSA ones. Thirdly and finally, where necessary the survey would allow to do analyses based on the subset of DSA-related projects. In the remainder of this paper, results are mostly presented for the entirety of the RAS cluster, except when looking at the particular standardization bodies and committees targeted, for which we isolated DSArelated projects.

A Web-based tool was used to build the survey and a link to the survey was distributed to the coordinators of the projects. The survey consisted of 51 questions and nested questions with possible responses ranging from yes/no answers, and single/multiple choice answers to open answers. The questionnaire was launched on 13 September and closed on 21 October 2013. Its response rate was of 85 percent; among the respondents, 64 percent represented STREP ${ }^{1}$ projects, followed by 23 percent of $\mathrm{IP}^{2}$ projects. The majority of the projects therefore has a funded budget ranging between $€ 2$ to $€ 4$ million. A large part of these projects is now reaching the end of the first year of their workplan. It should also be noted that a small percentage of respondents represented projects that not yet started their workplan. These projects were however deliberately also included in the survey, since a number of questions relate to what these projects have proposed in terms of standardization (committees targeted, effort dedicated etc.) and because the project coordinator is usually already aware of the limitations of his/her project regarding the accessibility to, the compatibility with and the potential impact on ongoing standardization activities within particular SDOs.

\footnotetext{
${ }^{1}$ Specific Targeted Research Projects are medium-sized focused research projects which typically run for one and a half to three years, with a small number of partners for which the European Commissions grants up to $€ 3$ million.

${ }^{2}$ Integrating Projects are medium to large-sized research projects with a duration between three to five years with a granted budget of up to $€ 25$ million.
} 


\section{SURVEY RESUltS}

\section{A. Contribution to standardization}

On average, projects considered standardization activities to be very important for the commercial success of their activities. Therefore, almost all projects $(90$ percent $)$ considered standardization activities in the proposal stage, dedicating specific work packages and tasks to these activities. However, contrasting to the high importance attributed to standardization, the projects surveyed planned to dedicate an average of 15 Person Months to standardization activities, which translates to an average of only 3 percent of the project's total effort (representing a variation between 0.4 and 6 percent). Among the standardization activities, half of the projects planned to contribute and influence/modify standardization, as well as monitor relevant standardization activities to ensure the system development is standard compliant.

Regarding current standardization activities, 68 percent of the projects are actively contributing to standardization, and most projects report that they are doing so according to initial proposal stage plans. However, one fifth of projects was not contributing to any Working Items, Projects or Groups at the time of survey, while one quarter contributes to one and another quarter of projects contributes to two of these SDO groups. Strikingly, $93 \%$ of projects report that academic institutions belong to their consortia are active in standardization, but only one third has SMEs participating; moreover, in two thirds of the cases, standardization is performed by consortium partners that were already involved in the standardization process before the project started.

Finally, it is worth mentioning that, while exactly half of the projects indicate that the results of their research will have regulatory implications -a finding which cannot be surprising, since spectrum is such a highly regulated domain- one fifth is not even monitoring relevant regulatory activities such as those taking place within CEPT and ITU.

\section{B. SDOs targeted}

Projects are contributing to a varied range of SDOs and Working Groups However, there is considerable overlap between the groups/projects targeted (with, logically, a strong emphasis on ETSI RRS and IEEE DySPAN), while at the same time some degree of coordination could result in a much wider impact, particularly for those projects only active in one group of one SDO.

\section{Collaboration}

With regard to collaboration with other projects, the vast majority of projects (91 percent) are open to synergies with other research projects and reciprocal support, e.g. to push a specific technology inside a Work Item/Project and co-signing of contributions. Similarly, 91 percent of projects would be interested in formulating a common response to regulatory consultation processes. The necessity of such collaboration can be derived from the fact that, at the time of the survey, 41 percent of projects identified important gaps in the current standardization state-of-the-art that could not be filled by these projects themselves. When asked for the reasons behind the existence of these gaps, a number of issues were mentioned, mostly related to a lack of resources, a lack of access to relevant standardization groups, the complexity of the specific standardization process/requirements of a certain group, and a time mismatch between project results and standardization timelines.

However, two thirds of projects did not yet identify other projects with which collaboration could be possible, and around 20 projects out of 22 have not (yet) engaged in such collaboration. When asked what could be the main advantages of inter-project collaboration, the responses can be grouped into the following categories: more impact because of increased size/strength/power; distribution of workload and resources; less and better focused contributed, sharing of expertise; better alignment between contributions due to preestablished coordination. Conversely, a number of potential bottlenecks were also identified by respondents: the wide scope of and diverging interests within the RAS cluster; the complexity of coordination (administratively, legally), the additional overhead and delay it may cause; the lack of communication between partners; and the strategic agenda of industrial partners, which may not be interested in joint standardization efforts per se.

There was no clear alignment between projects on which conditions should be fulfilled to enable standardization collaboration: some respondents think there is no need for formal agreements, while others responded that, at first, informal collaboration can take place and, in a later stage, collaboration needs to be formalized through, for example, an Non-Disclosure Agreement or formal liaison; others, however, responded that collaborations need to be formalized from the start.

Finally, with regard to the communication of standardization activities and results, 55 percent of the projects indicated that they are currently not communicating these in any way; However, most projects would be willing collaborate, for example via a common platform, on such communication and outreach activities.

\section{DISCUSSION AND CONCLUSION}

\section{A. Survey discussion}

Despite a number of initiatives taken to help bridge the gap between research and standardization, such a gap is still very much present in the Future Internet domain. In many cases, this prevents innovative research from resulting in successful commercial deployment.

As our survey shows, the current European Commission's Framework 7 projects on Radio Access and Spectrum Technologies, and the ones dealing with Dynamic Spectrum Access and Cognitive Radio in particular, are no exception to this general assessment. On the one hand, the projects surveyed mostly understand the importance of standardization for the industrial and regulatory acceptance and commercialization of the technologies they develop. As a consequence (and probably also because the European Commission insists on this), almost all projects include standardization as part of their proposal and dedicate specific 
tasks to it. On the other hand however, the planned effort invested in standardization only accounts for three percent of the total project budget on average, while standardization is typically a slow, complex and labour-intensive process (in the case of most major telecommunications SDOs requiring a regular attendance of and contribution to multiple day meetings in venues all over the world). Therefore, it may be no surprise that, while $68 \%$ of projects are actually contributing to standards, one fifth is not contributing to any group, and less prepared consortium partners (notably SMEs and those not previously involved in standardization) play a very limited role in the standardization process. The surveyed projects moreover considered the predominance of industry as one of the major bottlenecks for standardization coordination, since these industries often only represent their own, mutually conflicting interests. This, combined with the inherently limited lifetime of an FP7 project (only sometimes countered by the unstable practice of setting up "programmes" of consecutive projects), re-confirms the risk that the outcomes of FP7 projects on DSA and CR will not have sufficient impact on the standardization process. This risk is reinforced by an often occurring timing mismatch between project funding and project results on the one hand (end of funding just as results are mature enough to go into standardization), and between project results and standardization roadmap on the other hand (the limited window of opportunity to contribute to a specific standard).

In this regard, coordination of standardization efforts, as well as specific instruments to enable the standardization of research results beyond the project lifetime is desirable. For a number of reasons, there seems to be significant scope for such coordination. Firstly, the amount of SDOs and committees targets is broad, however there is significant overlap as well as complementarity between project activities (with a strong focus on ETSI RRS and IEEE DYSPAN for the CR related projects particularly analysed here). Not only could a concerted standardization effort result in an increased critical mass and a distribution of workload, it could also provide stronger, more focused and better aligned contributions (according to the projects themselves). Secondly, almost half of the projects identify gaps in standardization that cannot be filled in by the projects themselves. The required resources, the limited access to SDOs, the complexity of the process and the mentioned timing mismatch that are at the basis of this, are indeed difficult to be solved by the individual projects -or even individual project participants. Thirdly, $91 \%$ of projects are willing to collaborate in order to help resolve these gaps, but $86 \%$ has not yet done so. Fourthly and finally, more than half of the surveyed projects is currently not communicating externally about their standardization activities, while more than 90 percent is willing to do so.

\section{B. Proposals for DSA standardization coordination}

Following the conclusions of the survey, CRS-i evaluated the concrete research objectives and first standardization plans of various relevant RAS cluster projects, in order to assess where a coordinated approach could make sense and which concrete steps can be undertaken to increase the standardization impact of research outcomes. For this, first a thematic mapping was made which, is represented by Figure 1 below.

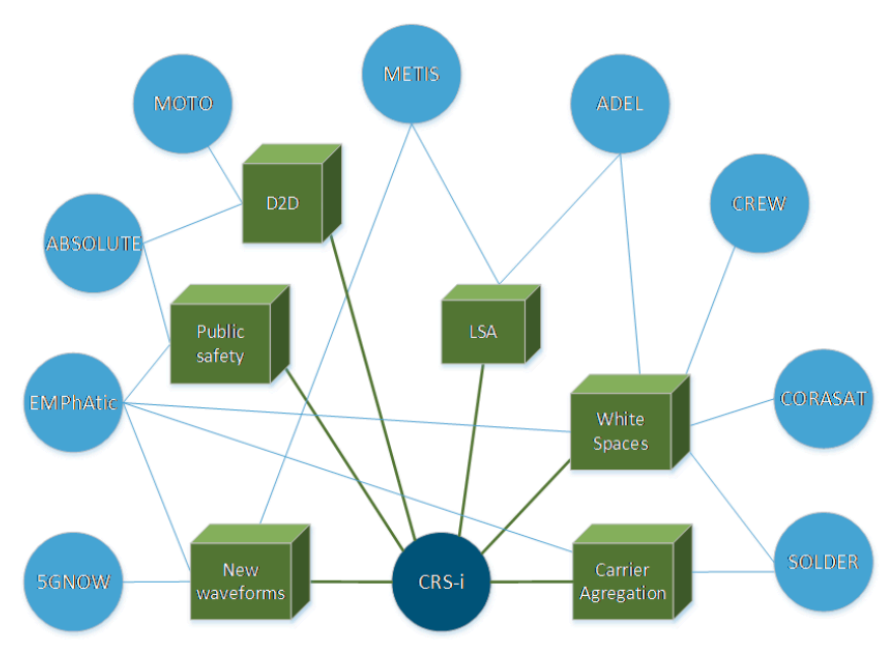

Figure 1: Relations between projects working on CR and DSA

The next step consisted in the mapping of concrete project research directions to target standards. Some, but certainly not all of these originate from the data provided by the projects themselves in the survey, since they are not always aware of the opportunities and ongoing activities in various SDOs. The mapping is shown in the figure below.

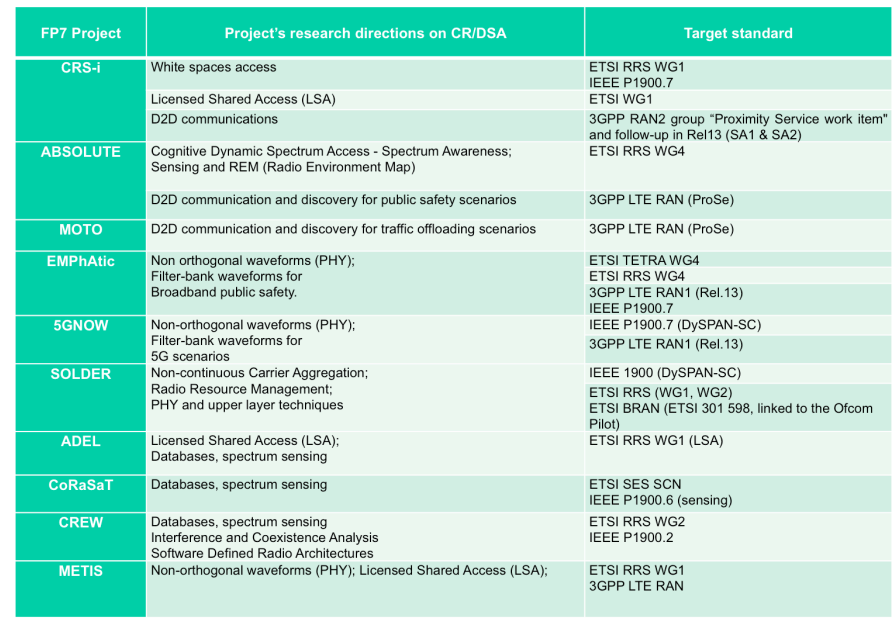

Figure 2: Overview of $C R$ and DSA related potential target standards for $E U$ funded research projects

The third step consisted of identifying opportunities for coordinated and concentrated efforts by various EU funded projects. Obviously, this is an ongoing process and subject to further interactions and approval of the projects. Specific attention is given to issues of confidentiality and IPR, which results in some actions being performed in the public domain (such as the ones listed below), while others are offered as bilateral consultancy services to individual projects (such as the standardization reports discussed further). By way of example, some of the actions currently under study by CRS-i are:

- Trigger a new Study Item in 3GPP on Non Orthogonal Waveforms (useful to EMPhAtic and 5GNOW, however 
the ideal timeframe of this action is beyond the timeframe of these projects);

- Contributions to 3GPP LTE RAN on D2D (ProSe): ABSOLUTE and MOTO, both lead by Thales, are involved, but extra support is needed, therefore a Task Force is considered;

- Contributions to LSA system architecture work in ETSI RRS WG1: here, CRS-I can support the ADEL project, however, it needs to be noted that the ADEL vision is beyond the current standardization work in this group (e.g. including sensing), whereas the ETSI priority is to finalize the architecture specifications with a practical/simple approach for LSA;

- Contributions to White Spaces technologies in IEEE DySPAN-SC: here, CRS-I and SOLDER may collaborate

- Contributions to ETSI RRS WG2 (SDR): here, SOLDER, EMPHATIC and CREW may collaborate;

- Contributions to ETSI RRS WG4: exploiting synergies among commercial, civil security and military domains (Objective $\mathrm{C}$ of the EC Mandate M512): CRS-i, ABSOLUTE and EMPhAtic may coordinate activities.

Besides these opportunities for coordinated standardization action, CRS-i has already actively promoted interactions between CRS-cluster projects towards an effective contribution to the work performed by SDOs, in particular the work done in ETSI RRS under the Standardization mandate for Reconfigurable Radio Systems (M/512). Very concretely,

- Work Items within SDOs related with the project scope have been identified, as well as what needs to be changed/included in the current standard draft to accommodate the project vision;

- tailored standardization reports for RAS cluster projects working on CR, DSA and coexistence issues have been produced. Each standardization report contains an overview of the standardization activities, the results of standardization meetings as well as opportunities for contributions to standards that fits in the project scope;

- $\quad$ projects updates have been presented by CRS-I on behalf of the projects in several SDO standardization meetings and organized dedicated meetings between standardization experts and the projects.

In conclusion, for $\mathrm{CR}$ and DSA, coordination between systems is of utmost importance. Without the outlining of procedures and requirements that are crystal clear for all stakeholders, the risk of interference is too high to generate the necessary trust with regulators and industry alike. These coordination requirements come on top of the classic economies of scale that are necessary in the capital-intensive telecommunications industry in order for products and services to be commercialized viably. If Europe is to play a role in the global standardization of $\mathrm{CR}$ and DSA both in terms of commercializing its own IPR and of safeguarding that Europespecific requirements are met by international standards, it must ensure a continuous, high-impact contribution to the most relevant committees and groups. It is highly unlikely that EU FP7 projects, due to their inherent limitations, can do this on their own. The Cognitive Radio Standardization Initiative is currently helping European projects to overcome these limitations, and thereby increasing impact of EU solutions on much needed, global CR and DSA standards.

\section{ACKNOWLEDGMENT}

The research leading to these results has received funding from the European Community's Seventh Framework Programme under grant agreement No. 318563 [CRS-i]. The authors wish to thank Paolo Aversano at iMinds for his help in setting up the on-line survey.

\section{REFERENCES}

[1] Radio Access and Spectrum Cluster, http://www.ict-ras.eu/

[2] http://sta.jrc.ec.europa.eu/pdf/corsa/Workshop_agenda_SoftwareDefined Radio_Cognitive Radio standardization.pdf

[3] European Commission (2010). Standardisation mandate to CEN, CENELEC and ETSI for Reconfigurable Radio Systems. http://ec.europa.eu/enterprise/standards policy/mandates/database/index. cfm? fuseaction $=$ search.detail\&id $=515 \#$

[4] A. Lorelli (2013). EC Mandate on RRS: status of activities and the way to move forward. Presentation given at the CRS-i/RAS Cluster Workshop "Radio Network Access Standardization: opportunities and challenges", Brussels, 22 October 2013

[5] B. Sales, E. Darmois, D. Papadimitriou, and D. Bourse (2012). A Systematic Approach for Closing the Research to Standardization Gap, Future Internet Assembly, Lecture Notes in Computer Science, Springer, Volume 7281, page 18-29

[6] D. Bourse (2011). Future Internet Assembly (FIA) Pre-standardization WG: Review of Objectives and Progresses. Future Internet Assembly, Poznan, Poland, 25 Ocotber 2011. http://www.futureinternet.eu/fileadmin/documents/poznan_documents/Session1_3 Standa rdisation/1-3-Bourse.pdf

[7] http://www.ict-crsi.eu/index.php/cluster-projects

[8] Didier Bourse (2011). Move FI Research into Practice Closing the Standardization Gap. Presentation at the EC Concertation meeting, Brussels, $\quad 10 \quad$ February 2011, available http://ec.europa.eu/information society/events/cf/fnc7/itemdisplay.cfm? $\mathrm{id}=5745$

[9] Report of the Future Networks 7th FP7 Concertation Plenary Meeting Brussels, 10 February 2011, http://cordis.europa.eu/fp7/ict/futurenetworks/documents/future-networks-standardisation-flagships. 